Cinémas

Revue d'études cinématographiques

Journal of Film Studies

\title{
Une voix en coulisse
}

\section{Suzanne Liandrat-Guigues}

Volume 3, numéro 1, automne 1992

Cinéma et Musicalité

URI : https://id.erudit.org/iderudit/1001177ar

DOI : https://doi.org/10.7202/1001177ar

Aller au sommaire du numéro

Éditeur(s)

Cinémas

ISSN

1181-6945 (imprimé)

1705-6500 (numérique)

Découvrir la revue

Citer cet article

Liandrat-Guigues, S. (1992). Une voix en coulisse. Cinémas, 3(1), 25-33.

https://doi.org/10.7202/1001177ar

\section{Résumé de l'article}

Cet article montre en quoi une idée de l'opéra organise la séquence d'ouverture de Senso à la Fenice de Venise, puis se dissémine dans l'oeuvre entière, à partir de la notion de " mélodrame », d'une thématique (celle du " trouvère ") et grâce à l'utilisation que fait Visconti de la voix. d'utilisation que vous pouvez consulter en ligne.

https://apropos.erudit.org/fr/usagers/politique-dutilisation/ 


\title{
Une voix en coulisse
}

\section{Suzanne Liandrat-Guigues}

\begin{abstract}
RÉSUMÉ
Cet article montre en quoi unc idée de l'opéra organise la séquence d'ouverture de Senso à la Fenice de Venise, puis se dissémine dans l'ceuvre entière, à partir de la notion de «mélodrame», d'une thématique (celle du «trouvère») et grâce à l'utilisation que lait Visconti de la voix.
\end{abstract}

\begin{abstract}
This article shows how an idea of opera organizes the opening sequence in Senso in the Fenice theatre of Venice, and then extends through the entire work, using the notion of "melodrama", the theme of the "troubadour" and the use Visconti makes of the voice.
\end{abstract}

L'époque de Senso coïncide avec le moment des créations de Visconti à la Scala de Milan. À La Vestale de Spontini (1954) succèdent en 1955 La Somnambule de Bellini et La Traviata de Verdi avec Maria Callas. Cette expérience lyrique, nouvelle pour le réalisateur, explique sans doute que le film soit si fortement marqué de l'empreinte de l'opéra. Si Visconti a tourné 14 longs métrages, sa théâtrographie est beaucoup plus importante et compte 18 mises en scène d'opéra.

Considérer Senso comme un opéra cinématographique n'est pas nouveau, mais pourquoi choisir en 1953 le modèle de l'opéra alors même que sa disparition daterait du Turandot de Puccini (1924) ou que, comme le rappelle Bernard Dort, «depuis 1920 environ, la grande majorité du public cultivé s'en tient là : l'opéra n'est plus, comme le disait alors Brecht, qu'un "genre culinaire"» (p. 1)? 
Mort de l'opéra ou crise de l'opéra, cependant, dit Philippe-Joseph Salazar :

(...) il suffit de jeter un oeil aux saisons des opéras des capitales pour voir qu'il vit. Mieux il se survit, mais avec quel luxe! (...) L'opéra se paie le luxe de ne rien créer de neul; au contraire, l'opéra déterre des œuvres avortées en leur temps, met à jour des manuscrits demeurés lettre morte; brel, loin de laire répondre sa perpétuation de son renouvellement, l'opéra vit d'un passé oublié (p. 8).

L'opéra est donc une forme de création morte au moment où Visconti entreprend ce film. Le genre est entré dans l'ère des réinterprétations et l'on parlera de la «Salomé de Wieland Wagner» ou de la «Traviata de Visconti»... La mise en scène de spectacle lyrique par Visconti est une interprétation "philologique» d'œuvres issues de telle ou telle période historique. Ce point de vue peut être retourné en avançant l'idée que voulant mettre en scène au cinéma une page de l'histoire italienne située au moment de la pleine gloire de Verdi, il ait imaginé tout naturellement d'introduire ce trait historique dans l'univers du film. La nouvelle de Camillo Boito, frère d'Arrigo Boito (collaborateur de Verdi), le Carnet secret de la comtesse Livia dont s'inspire le scénario, ne fait aucune place à l'opéra. À l'étonnante «piscine» imaginée par Boito pour lieu de la rencontre entre Livia Serpieri et l'officier autrichien succède la «baignoire» de laquelle la comtesse assiste à l'altercation entre Franz Mahler et son cousin Ussoni; il est vrai que la salle théâtrale a souvent été comparée à un aquarium...

L'évolution et la crise de l'opéra miment la mort d'une société tandis que le film présente une page de l'histoire italienne qui montre le point de fracture et de décadence d'une classe sociale, la disparition d'un monde. $\mathrm{Ce}$ thème qui court dans l'œuvre cinématographique de Visconti est dédoublé dans l'usage qu'il fait d'une œuvre du répertoire classique des opéras. Des deux côtés, la mort. Le premier film du réalisateur à ouvrir la longue série des histoires de familles en voie de disparition fait place à un opéra, Le Trouvère, dont le livret est justement une histoire de famille qui se détruit puisque le comte Luna finit par tuer son propre frère. Le lien intime entre une forme de représentation comme l'opéra et le propos du film expliquerait le choix de Visconti. Selon Youssef Ishaghpour :

Senso révèle le fondement historique et esthétique de son style. L'opéra y apparaît clairement comme une superstructure, c'est l'imaginaire d'un vécu prosaïque qui s'y révèle et s'y voile à la fois. Le rapport de ce vécu à cet imaginaire, de la salle à la scène, de l'Histoire à sa vision idéologique, lait de l'opéra bien autre chose qu'une ouverture un peu pittoresque ou qu'un lieu de manifestation politique. "C'est une sortc de clé» a dit Visconti, "pour les Italiens 
de l'époque, crier "Vive Verdi" ne signifiait pas seulement "Vive Emmanuel, roi d'Italic", cela signiliait aussi "Vive Giuseppe Verdi", cela signifiait qu'on aimait une musique déterminée, et c'est après tout une question de siècle el de situation» (p. 79).

Senso débute par la représentation sur la scène de la Fenice à Venise de la fin de l'acte trois du Trouvère de Verdi. Le film donne à entendre et à voir en totalité la fin de l'acte trois jusqu'aux applaudissements; mais on ne peut savoir à quoi correspond exactement le temps de l'entracte. Lorsque reprend l'acte quatre, la voix des chanteurs s'élève tant que l'action se déroule dans la salle de la Fenice.

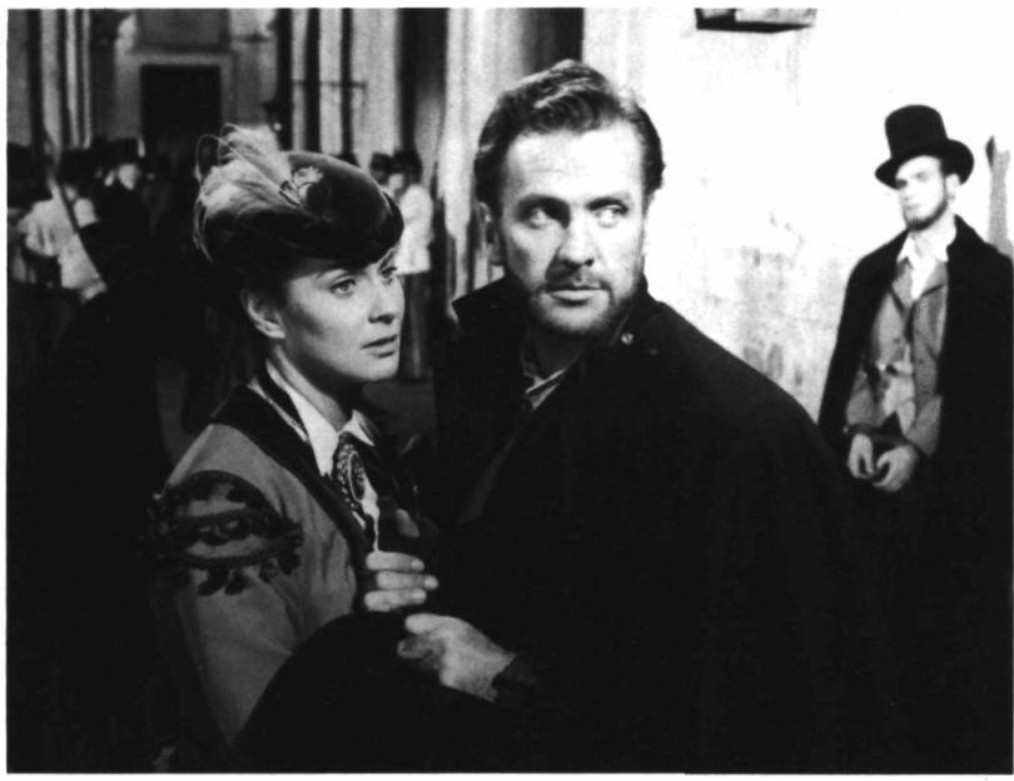

Senso de Luchino Visconti (1953)

Coll. Cinémathèque québécoise

L'intrigue de Senso reste affectée par l'épisode initial. Cette première séquence constitue la cellule de base d'une composition cyclique dont les éléments, irradiant d'autres séquences du film, diffractent la composante théâtrale. Il en est ainsi des plongées 
nettement marquées que l'on trouve à divers moments du film et qui correspondent à la vision dominante des spectateurs des loges ou du poulailler súr l'espace scénique du Trouvère. Cette plongée met en exergue la prouesse du chanteur interprétant la cabalette de Manrico «Di quella pira». Elle signale un moment hautement théâtral et correspond visuellement à une attitude d'écoute de la salle qui a reconnu les signes annonciateurs du morceau de bravoure (changement d'orchestration, montée à l'avant-scène du chanteur). Lorsqu'une plongée brutale reparaît dans un autre passage du film, elle rappelle la première et sous-entend la théâtralité du décor, des postures ou des relations entre les personnages. Il en est de même de l'architecture qui, de l'ensemble aux détails, reprend la forme de l'arcature de la loge et de la scène de l'opéra ou du motif récurrent du rideau rouge, qui évoque les rideaux de scène de la Fenice, ou de l'éclairage bleu qui inonde la tour au pied de laquelle chante Leonora au début de l'acte quatre1. Le dernier épisode du film s'achève sur le mur de Vérone devant lequel est fusillé Mahler et renvoie à la muraille de l'acte quatre, derrière laquelle est condamné Manrico. De la Fenice à Vérone, l'espace se clôt et fait retour à la scène théâtrale initiale. La couleur bleue envahit les murs devant lesquels erre Livia après avoir provoqué l'arrestation de Mahler. Comme les héroïnes mélodramatiques, elle entame un air de la folie. Cette sortie théâtrale vers les coulisses du décor vaut bien un contre-ut : la prouesse accomplie par Alida Valli hurlant le nom de Franz lui coûta quatre cordes vocales.

L'empreinte du théâtre lyrique a fortement marqué Senso en raison du traitement des voix. L'axiome fondamental de l'opéra, pour Michel Poizat, est «la prééminence absolue de la dimension vocale» (p. 202). Il est mis en évidence par le décalage systématisé entre voix et personnage :

Cela veut dire que ce ne sont pas les caractéristiques du personnage qui vont déterminer la matière musicale (...) c'est à l'inverse aussi paradoxal que cela puisse paraître, le désir, le parcours de jouissance vocale que l'on vise qui va déterminer fondamentalement la nature du personnage choisi, celui-ci devient ainsi en quelque sorte, le porteparole, ou plus exactement l'enveloppe manifeste du désir sousjacent lié à la voix. (...) La voix n'est pas l'expression d'un texte, c'est le texte qui est l'expression de la voix. (...) Ce n'est pas parce qu'une logique du livret amènc la femme à la mort qu'elle crie en cet instant; les conditions dramatiques nécessaires à l'apparition du cri sont créées parce qu'une logique de la jouissance vocale est à l'œuvre et "pousse au cri» (...). Cela est si vrai que fréquemment les supports anecdotiques délaillent (incohérence ou invraisemblance totale du livret) sans que la composition vocale en pâtisse (pp. 202203) 2 . 
La détermination des rapports voix-personnage découle fondamentalement de ce principe et fait des propriétés du rôle (ses paroles, sa situation) un effet d'après-coup de ce qui se joue au niveau de la voix. À l'opéra, la musique et la voix nous parlent profondément des êtres qui s'agitent sur la scène. Ainsi s'explique mieux l'impression que fait naître le film dans la séquence de la Fenice. Un lien fort s'établit entre Livia, Mahler ou Ussoni et les personnages de Verdi. La voix apparaît comme première et le comportement mélodramatique qu'auront ces êtres en découle.

Chacun se rappelle la procédure par laquelle Visconti déplace le spectacle de la scène vers la salle et qui transforme la salle, les loges en lieux théâtraux. Franco Zeffirelli a rapporté que les indications de mise en scène dominantes de Visconti furent :
(...) l'angle de vision donné sur la scène et la salle ensemble (la représentation étant vue de profil) par la position des loges d'avant- scène à la Fenice comme à la Scala, où Visconti en aurait eu l'intuition pour son film. (...) Ainsi dans Senso «le point de vue de l'avant-scène» introduit noblement le dévoilement de l'envers et de l'endroit de toutes choses, concourant au développement du drame (Acher, p. 5).

Le rôle du contre-ut que la tradition impose au ténor est de souligner cet effet puisqu'il est en quelque sorte le signal de l'insurrection italienne. Cet exploit vocal constitue une déviation par rapport à la partition de Verdi qui ne faisait pas appel à cet aigu. Il engendre une autre forme de détournement puisque désormais le spectacle est dans la salle ${ }^{3}$. Si le contre-ut conduit l'action sur la scène comme dans la salle, c'est qu'il affecte des lieux différents. Il a, comme on l'a souvent observé, la curieuse propriété de continuer à résonner dans la salle alors que le chanteur est reparti dans la coulisse. C'est que la voix en se répandant dans la Fenice peut occuper un emplacement impossible à localiser avec exactitude. Aussi l'espace tout entier devient-il musical.

Michel Leiris écrit :

Le chant - variété hautement stylisée de la parole - s'avère organiquement lié aux éléments immédiatement «théâtraux» que sont les positions respectives des personnages, leurs déplacements, leurs apparitions et leurs éclipses (p. 10).

Pour l'écrivain, sont déterminantes les positions respectives des interprètes avec la scène et leur répartition sur des plans spatiaux $\mathrm{et}$ vocaux bien distincts. Il en est ainsi lorsque Livia Serpieri et Franz Mahler sont réunis dans la loge du Gouverneur et que la cantatrice interprète l'aria de Leonora «D'amor sull'ali rosee vanne sospir dolente» au début de l'acte quatre. Les positions respectives des 
deux personnages et de la cantatrice vus dans un miroir, qui répète dans son ornementation les volutes du rideau de scène, constituent un nouvel espace lyrique. Fragmenté par les cadrages, dupliqué par les jeux de miroir, cet espace sonore est fondé sur les séparations et les distinctions des plans vocaux. En effet, lorsque Mahler rejoint la loge où se trouve Livia, elle est installée au fond, dos à la scène, et tournant la tête à l'arrivant. Elle est alors orientée face à la cantatrice qui se trouve dans la profondeur de champ, séparant Livia et Franz. Un peu plus tard, lorsque débute l'aria, Livia et Leonora sont presque alignées et Mahler est assis auprès de la comtesse. De ces rapprochements et de ces écarts entre les protagonistes naît l'illusion que la loge et la scène sont un même espace, confusion semblable à celle éprouvée par la salle à la fin de l'acte trois quand l'appel aux armes lancé par Manrico enflamme ses sentiments patriotiques.

L'espace de l'opéra repose sur les relations entre les voix et les lieux qu'elles occupent diversement. À la division attendue entre chanteurs et instrumentistes, les uns sur scène, les autres dans la fosse d'orchestre, se superpose une organisation plus complexe propre à l'opéra. «Dans ce théâtre-là, l'œuvre paraît avoir pour toute spéciale raison d'être de susciter l'éclosion d'un espace intensément sensible» (Leiris, p. 10). De là vient que la voix de Livia ou celle de Mahler ne semblent pas hétérogènes à celle de la cantatrice.

Comme l'observe Michel Leiris, le célèbre «Miserere» du dernier acte du Trouvère repose sur l'utilisation des «moyens par excellence de l'opéra, qui n'est pas seulement du théâtre en musique mais de la musique dans un espace théâtral» (p. 8). Il évoque ainsi ce trio :

À la plainte pathétique du soprano placé sur le devant de la scène s'associent - en une étonnante mosaïque de styles - le chant brillant du ténor qui, censément enfermé dans les murs d'une prison, se tient dans la coulisse et les clameurs ou bourdonnements d'un chœur de caractère religieux, émanant lui aussi d'une source cachée mais celle-là impossible à exactement localiser (p. 7).

Il est remarquable que Visconti ait arrêté la séquence de la Fenice juste avant que ne commence le «Miserere». Cette diffraction de l'espace sonore, règle d'or de l'opéra selon $\mathbf{M}$. Leiris, ne se réalise pas au moment où elle est le plus attendue, c'est-à-dire dans le célèbre trio constitué de Leonora, de Manrico et du chœur des moines. Il semble que Visconti (jouant peut-être avec le savoir du spectateur) fasse du reste du film l'actualisation de cette procédure. Différée, la procédure sera en fait diffractée 
dans l'ensemble de l'œuvre selon un principe qui est la multiplication des éléments musicaux.

La voix de la narratrice s'inscrit dans cet eșpace sonore. Le statut romanesque du narrateur contant sa propre histoire (comme c'est le cas dans la nouvelle de Boito) ne peut se concevoir à l'opéra ou au théâtre sous cette forme. Visconti fait de cette voix "off» une véritable voix lyrique qui, par sa mobilité, ses apparitions (12 occurrences dans le film) et ses disparitions, façonne théâtralement les scènes qu'elle accompagne. Cette voix est entendue pour la première fois dans la séquence de la Fenice, pendant l'entracte, alors que Livia quitte la loge et s'enfonce dans un corridor. La comparaison avec la sortie en coulisse s'impose. Mais tout aussi bien l'interpénétration des espaces, l'envers et l'endroit se retournant, car au bout de ce corridor Livia débouche sur le foyer de l'opéra où elle rejoint son cousin Ussoni, qui bout d'une fièvre révolutionnaire digne d'un héros romantique.

Après la séquence de la Fenice, la voix réapparaît alors que Livia est dans la prison où se trouvent rassemblés les patriotes italiens. Lors de sa troisième manifestation, dans ce même lieu, elle est associée à la musique de Bruckner. Le film propose 24 utilisations des thèmes du premier ou du deuxième mouvement de la Symphonie no 7. Dans le générique, la musique de Bruckner assure la fonction de "commentaire musical» (expression que Visconti reprendra à propos de la pièce de César Franck dans Sandra). La voix de la narratrice et la musique de Bruckner se succèdent généralement, mais il arrive que les deux se superposent ou que la musique s'élève au-dessus des échanges amoureux entre Livia et Franz. Le son des cloches n'est pas absent dans la chambre où Livia attend vainement Franz. Peu après, un fondu enchaîné la conduit à la chambrée des officiers qui ont entonné un lied de Schubert (appartenant au Voyage d'hiver). Tous ces éléments composent entre eux pour que se développe, en dehors de la scène de l'opéra, un lyrisme fondé sur les divisions sonores et spatiales. En outre, la voix de la narratrice surgit toujours sur un décor associé à une vaste perspective (couloir, galerie, escalier, quai ou place de Venise, portique à colonnes d'Aldeno, enfilade de portes, rue ou perspective d'un tableau). Elle est perçue comme une voix de la coulisse instituant un arrière-plan, une profondeur, par rapport à la scène montrée immédiatement avant ou après.

Le choix de l'opéra par Visconti en 1953 pourrait donc se justifier, entre autres, par la possibilité qu'offre ce moyen d'expression d'une voix venant d'un espace autre. Voix et espace qui, dans le film, se convertissent aussitôt. D'une part la conversion est celle du passage d'un espace dans un autre; les personnages, l'intrigue ne nous paraissent jamais tant 
mélodramatiques que lorsque l'espace présent à l'écran fusionne par l'imaginaire avec l'espace du spectacle lyrique. D'autre part la voix étant par excellence ce qui se perd, diffuse ou retentit, elle favorise la création d'un hors-champ temporel, toujours difficile à mettre en œuvre au cinéma alors que dans la nouvelle de Boito, la voix de la narratrice était narrativement localisable «16 ans après une aventure arrivée à Venise». Depuis quel temps s'exprime la comtesse du film reste tout à fait indécidable. Selon Youssef Ishaghpour :

(...) le style représentatif, ainsi appelait-on l'opéra à sa naissance, c'est la représentation devenue ostentatoire, l'art de l'effet, de l'apparence (...). L'opéra par le jeu de ses réinterprétations est devenu une forme éternellement répétitive au moment où Visconti entreprend son film, lui-même ajoutera un tour à cette spirale. Recommencement et mort du temps. De fait Livia Serpieri avoue qu'à Venise "le temps n'existait plus". La passion de Livia, le mélodrame, est la négation du temps (p. 73).

Université de Paris III

\section{NOTES}

1 Pour une étude de ces reprises, voir mon article «Dans l'écartement du rideau rouge», Studio 43 (1990) pp. 75-87.

2 Ce que prouve à l'évidence le discours narrativement confus que tient Azucena à son «fils» Manrico à l'acte deux.

3 L'expression «le spectacle est dans la salle» renvoic bien évidemment aux relations que le public de l'époque établit entre l'opéra de Verdi et l'histoire nationale italienne. Michèle Lagny, dans Senso (Paris : Nathan, 1992), observe à propos du contre-ut qui «déclenche les cris de Viva l'Italia, Viva La Marmora et même Viva Verdi» que «l'incident ne semble pas s'être produit en mai 1866 à la Fenice, mais d'autres du même genre se sont renouvelés à plusieurs reprises, aussi bien à la Scala de Milan en 1842 lors de la représentation de Nabucco que dans bien d'autres cas, et pas seulement pour des ceuvres de Verdi (par exemple, quand la salle reprenait en chœur les imprécations de Norma, "Guerra, guerra")" (p. 59). On peut ajouter qu'en mars 1844, à l'occasion de la représentation d'Hernani, éclatèrent de semblables manifestations et qu'Attila souleva à la Fenice les cris de A noi l'Italia, a noi! Sur Senso et le Risorgimento, voir le texte de Leger Grindon, «Risorgimento History and screen Spectacle: Visconti's Senso», in Robert Sklar et Charles Musser (ed.), Resisting Images, Essays on Cinema and History (Philadelphia : Temple University Press) pp. 225-250. En outre, l'expression «le spectacle est dans la salle» peut s'entendre indépendamment de la conjonction historique italienne, dans cette dimension propre aux comportements spectatoriels de l'époque où le public n'observait pas le recueillement qu'on lui connaît aujourd'hui. 


\section{OUVRAGES CITÉS}

Acher, Willy. «Pour saluer Visconti». Cahiers du cinéma 57 (mars 1956).

Dort, Bernard. «Le théâtre par excellence». L'Arc 27 (1965 et rééd. 1990).

Ishaghpour, Youssef. Visconti, Le sens et l'image. Paris : éd. de la Différence, 1984.

Leiris, Michel. «L'opéra, musique en action». L'Arc 27 (1965) pp. 7-12. Article repris dans Brisées. Paris : Mercure de France, 1966, pp. 279-285.

Poizat, Michel. L'Opéra ou le cri de l'ange. Paris : Métailié, 1986.

Salazar, Philippe-Joseph. Idéologies de l'opéra. Paris : PUF, 1980. 